\title{
Research on motivation and iconicity of Chinese grammar
}

\author{
Zeng Wang
}

\author{
Liaoning Jianzhu Vocational University, Liaoyang, 111000, China
}

Keywords: Chinese grammar; Motivation; Iconicity

\begin{abstract}
With economic development of China and research on long history and culture of the country, learning Chinese language gradually becomes fashionable and popular. Research on Chinese grammar is a difficult and key point. This thesis mainly focuses on discussing motivation and iconicity of Chinese grammar.

In China's long history, the ancients started motivation and iconicity of language early. For instance, the book Origin of Chinese Characters written by Xu Shen a scholar in the Eastern Han Dynasty carries out specific research and thinking on this aspect. Motivation and iconicity of Chinese grammar are of great significance for linguistics and also have much blank space for research and exploration. Especially, they are of practical and positive importance in teaching Chinese as a foreign language (TCFL) and speech recognition technology of computer.
\end{abstract}

\section{Motivation of Chinese grammar}

Usually, motivation of Chinese grammar is a motivation form that can be demonstrated and is non-random in linguistic form and significance, which reflects relationship of meaning. Generation of the term represents people's cognition about language and thinking reaches a new height and gets logical development planning. Research on grammar has been looking for and trying to find out motivation road of language itself. Especially introduction to computer and development of future speech recognition technology are essential for analysis and research on motivation of grammar. In accordance with analysis, it can be found that motivation is reflected by different levels of language: (1) the level of words; (2) the level of semantics and (3) the level of grammar. At the level of words, we may find motivation via compound words. For example, 'see' is an effect entirety of an action and a result. Besides, the word 'stand' shows there is motivation between form and meaning. At the level of semantics, conceptual conversion is also a form of motivation sometimes. For instance, 'the first hand' and 'half a bottle of water' are a form of conceptual conversion. At the level of grammar, motivation of Chinese grammar generally refers to reasons and basis on which all kinds and levels of syntactic entities constitute combinations and laws [1]. We will use examples to analyze motivation of Chinese grammar in the aspects of word order, semantics and grammar. Word order refers to the order in which morphemes and words are combined in language. According to Shi Shuzhi's article, it can be found that word order is the most important expression method of grammar in Chinese and there must be different expression functions in different word orders.

Importance of Chinese word order often contains the following three aspects that correlate with one another. Firstly, other languages use morphology or grammar signs to express grammatical meanings, while Chinese usually uses the form of word order for expression. Secondly, for the same group of words, Chinese often utilizes various changes in word order to express different meticulous and rich grammatical meanings. Thirdly, Chinese expresses leading grammatical relation of people's language sentences via word order. For instance, verbs follow subjects, objects follow verbs and modifiers are always in front of head-words of predicates. Hence, we may know word order of Chinese is its description about objective iconicity. Chinese often uses time as an order to describe the objective world more directly, more commonly and more consistently compared with other languages.

Let us have a look at the following example sentences: (1) time is used as an order, including 'yesterday, today and tomorrow'. By virtue of this simple sentence, a statement using time as an order can be obtained. Similarly, there are other example sentences, such as 'set questions to test students', 'he will go to America after meal' and 'go to Shanghai by train'. In addition, examples 
about compound sentences are shown as follows: 'You may go to toilet before having dinner', 'Let's go to the park after getting up tomorrow', 'He is ill, so he did not have training today' and 'Although the orphan does not have parents, all of us help her'. All of these example sentences use time sequence as a law. Thus, we may find Chinese grammar has the independent law that it uses time sequence as a principle. (2) For range of space and time, we should express larger space-time ranges first, such as 'June 1, 1997' and 'Pudong International Airport, Shanghai, China'. Besides, we should talk things with higher positions first and then the ones with lower positions, such as 'heaven, earth, country, ancestor and sage', 'province, city and county' and 'elder sister and younger sister'. (3) Entirety is prior to parts, such as 'in museum in the center of the square', 'newsstand near the road' and 'he carries a cat'. (4) Modifiers are prior to central words, such as 'dear country', 'walk slowly', 'I am tired' and 'red flower'. (5) Conditional sentences are prior to main sentences, for example, 'As long as we learn grammar well, we can speak Chinese well'. (6) Relativity is prior to absoluteness, such as 'eve of this new year' and 'the capital Beijing'. (7) Known situations are prior to unknown ones, such as 'How much are your peanuts' and 'Running race is canceled because of snowing'.

During research and analysis, we also find word groups and word have parallel relationship, which is also an expressive form of motivation of grammar. For instance, we can find internal unification and parallel nature of Chinese grammar and sentence in the following examples, such as 'first, second, only, often, desk, wood, all sorts of, womanishly fussy, in a fog, badly battered, talk one by one and one class by one class'. According to a large number of example sentences, we can know Chinese grammar has its laws and motivation. The motivation can be comprehended via analyzing Chinese grammar. In addition, it can be found that Chinese has high unification and balanced relationship. After mastering these laws, we may find secret of Chinese and it also has positive effect on improvement TCFL quality.

\section{Iconicity of Chinese grammar}

It was Charles Sanders Peiree that put forward iconicity of grammar for the first time. Generally, iconicity signs are divided into three types: (1) Image, which involves signs and objects only have similarities in some attributes simply, for instance, photos; (2) diagram, which involves signs and objects have similarities in structural relationship, for example, maps; and (3) metaphor, which refers to signs used to reflect features of signified objects via parallelity between this object and that object. Later, Givon put forward three laws of iconicity further, including (1) quantity iconicity; (2) distance iconicity and (3) order iconicity. The iconicity in the aspect of quantity has wide extension. Connotation of its importance and predictability is quite complicated. It mainly involves recurrence phenomena of complexity, morphology and syntax o language in the process of constitution. For example, we may find complicated iconicity in the aspect of syntax is one of the types of iconicity according to examples like 'I am tired because of washing clothes' and 'Li feels anxious after waiting for express for a long time'. Based on examples like 'every family', 'beautiful' and 'more or less', we can find they stand for a kind of iconicity that is composed of repetition of quantifiers. Besides, in accordance with examples, such as 'You should think about yourself', 'I will watch videos and listen to CD tonight', 'You may try on this clothes to judge whether it is appropriate for you' and 'blinking', it can be found that they represent a kind of iconicity which is composed of repetition of monosyllabic verbs. Considering which verbs can be repeated while which ones cannot be repeated, we should judge them by thinking about whether actions can be extended and repeated. This is complicated, so we will not talk much about this here. Iconicity of distance is mainly reflected by combination of noun phrases and the word '的'. Detailed examples are shown as follows:

A1

$$
\text { “红花 }
$$

“暖水
A2

红的花

暖的水
B

血红的花”

暖暖的水” 
“臭衣服臭的衣服臭臭的衣服”

“好书好的书很好的书”

Professor Zhang Min studies and analyzes them, thinking that structural meanings of modifier-head construction reflects differences in distance of concepts when the two kinds of adjectives act as modifiers and the two forms, i.e., with the word '的' and without the word, also have differences. To sum up, we may find $\mathrm{A} 1<\mathrm{A} 2<\mathrm{B}$. For instance, 'red' and 'flower' are not separated in A1, but they are separated by the word '的' in A2. In addition to '的', another morpheme 'blood (血)' is added to B. According to the three patterns, we may know the degree to which modifiers and central words are combined, and language distance of the form with close combination is shorter compared with that of the one with loose combination. For instance,

A1

A2

$\mathrm{B}$

$$
\begin{aligned}
& \text { “*好一首诗 *好的一首诗 好好的一首诗” } \\
& \text { “*好那首诗 ?好的那本书 好好的那首诗” } \\
& \text { “*脏一条裤子 *脏的一条裤子 脏兮兮的一条裤子” } \\
& \text { “*脏那条裤子 脏的那条裤子 脏兮兮的条裤子” }
\end{aligned}
$$

In the forgoing example, quantity components cannot be added to A1 completely since they are combined closely and their formal distance is the shortest. A2 comes second. In detail, definite quantity components cannot be added but definite components can be inserted. The formal distance of $\mathrm{B}$ is the largest among the three, i.e., both indefinite and definite components can be added basically. Semantic difference implied by different noun phrases and the word '的' can be reflected by the following matrix relationship.

Semantic difference:

$\begin{array}{ccc}\text { Appellation } & \text { Classification } & \text { Predication } \\ \mathrm{A} 1+ & + & - \\ \mathrm{A} 2- & + & + \\ \mathrm{B}- & - & -\end{array}$

'Appellation' refers to 'namable nature', 'classification' means their functions and 'predication' denotes an assertion with weak connotation of the logical relationship containing A1 and A2 concepts. Thus, we draw the following conclusion.

The structure of category A has classification. The structure of category A1 owns appellation, and the structure of category A1 and B has predication. Predication of the latter is higher than that of the former. All of these are caused by conceptual distance such an agent. In the foregoing example sentences, '的' in category B must appear. Thus, concealment about the word '的' does not exist. It is necessary to analyze formation conditions of A1 and A2. If the word '的', which serves as a modifier-head sign, is concealed, background will be hidden. For instance, 'red' acts as a basis on which 'paper' is classified in 'red paper and blood-red paper' (“红纸 and 血红的纸”), the word '的' is implicit in the former word while it is explicit in the latter one. Classification indicators in the latter have classification meanings with high degree and high stability [2]. Linguists also classify possessive relationship into two kinds, i.e., 'transferable' and 'non- transferable'. The former represents an owner and belongings have inseparable relationship, just like the relation between human and his body. However, the latter refers to the possessive relationship that can be transferred, such as people's treasure and articles etc. Many scholars find the interesting phenomenon that non- transferable relationship usually is closer than transferable relationship. This relationship also indicates iconicity of language distance. For example, the reason why the iconicity 
of the order, i.e., some parts of body $>$ domestic relation $>$ common articles, is generated mainly lies in time. Time sequence is one of the basic structures and opinions of people's cognition as well, which reflects the reason why dominant order iconicity of linguistic forms is generated. According to this section, we analyze quantity iconicity, distance iconicity and order iconicity as well as the reason why such kinds of iconicity are caused.

\section{Significance of research and analysis of motivation and iconicity of Chinese grammar for TCFL}

Although there are many disputes on effect and significance of grammar teaching, all schools of language teaching value grammar teaching very much and need carry out profound research. During the long period of traditional language teaching, grammar teaching has been holding a central position all the time. Grammar teaching covers main content of foreign language teaching. Ancient and traditional language teaching is just composed of vocabulary teaching and grammar teaching. Modern language teaching changes previous patterns, emphasizes oral teaching comes first and treats sentences as a basic teaching mode. Language habit is developed by imitation, recitation and practice. Though sentence grammar is not analyzed directly, it is advocated that we should draw essential conclusions about grammar laws and find grammar laws via conclusion in order to obtain improvement at a higher level. Currently, some people advocate that grammar teaching should be weakened for TCFL. The reason why this wrong cognition is generated is that teachers do not realize features and laws of Chinese really. By applying analysis about motivation and iconicity of grammar, we may draw inferences about other cases from one instance in teaching and courses. By virtue of grammar laws, students can master a number of vocabularies, sentences and their grammar rapidly in learning via similar examples and can learn language better and more comprehensively. Research and analysis about motivation and iconicity of grammar also have positive effect on textbook compilation. By analyzing motivation and iconicity of grammar, teachers may make textbooks more practical, more scientific and more purposeful [3]. The motivation and iconicity that Chinese grammar itself has is not a simple form of language but a form which reflects Han nationality's thinking model and Chinese culture at a deep level. When you learn internal content deeply, motivation and iconicity of Chinese grammar reflect their value. Thus, for TCFL, research and analysis about explanation about Chinese grammar are a higher requirement. Explaining Chinese grammar reasonably in teaching activities, especially analyzing its motivation and iconicity, is a higher requirement for improvement in Chinese teaching. For many linguistic enthusiasts, research and analysis about motivation and iconicity of Chinese grammar are essential objects of study to a larger extent.

\section{Conclusion}

This thesis analyzes motivation and iconicity of Chinese grammar as well as laws about generation of motivation and iconicity from the perspective of grammar. Finally, it states significance and effect of motivation and iconicity of Chinese grammar on TCFL. Statement of this thesis is theoretical discussion to a large extent, so its specific effect need be practiced and verified by scholars in practical application and analysis further. At the same time, when we explain motivation of Chinese grammar, it does not mean there is no non-motivation in Chinese grammar. Readers need analyze and study this aspect further.

\section{Reference:}

[1] Lu Weizhong. Research overview about iconicity of language [J]. Foreign Language Teaching and Research (bimonthly), 2011(6):840-849.

[2] Li Xiaoqi, Zhang Xin. Research on grammar teaching in teaching of Chinese as a foreign language under new situations [J]. Chinese Language Learning, 2010 (1):77-87. 
[3] Li Baogui. Ontology research on teaching of Chinese as a foreign language and Chinese [J]. Beijing: Peking University Press, 2005:2-13. 